\title{
Events with an isolated lepton and missing transverse momentum and measurement of $W$ production at HERA
}

\section{The $\mathrm{H} 1$ and ZEUS collaborations}

\section{E-mail: tobias.haas@desy.de}

ABSTRACT: A search for events containing an isolated electron or muon and missing transverse momentum produced in $e^{ \pm} p$ collisions is performed with the H1 and ZEUS detectors at HERA. The data were taken in the period 1994-2007 and correspond to an integrated luminosity of $0.98 \mathrm{fb}^{-1}$. The observed event yields are in good overall agreement with the Standard Model prediction, which is dominated by single $W$ production. In the $e^{+} p$ data, at large hadronic transverse momentum $P_{T}^{X}>25 \mathrm{GeV}$, a total of 23 events are observed compared to a prediction of $14.0 \pm 1.9$. The total single $W$ boson production cross section is measured as $1.06 \pm 0.16$ (stat.) \pm 0.07 (sys.) pb, in agreement with an Standard Model (SM) expectation of $1.26 \pm 0.19 \mathrm{pb}$.

KEYWORDS: Lepton-Nucleon Scattering

ArXIV EPrINT: 0911.0858 
The H1 and ZEUS collaborations

F.D. Aaron, ${ }^{13, a 8}$ H. Abramowicz,${ }^{72, a 36}$ I. Abt,${ }^{57}$ L. Adamczyk, ${ }^{19}$ M. Adamus, ${ }^{84}$ M. Aldaya Martin, ${ }^{31}$ C. Alexa, ${ }^{13}$ V. Andreev,${ }^{54}$ S. Antonelli, ${ }^{9}$ P. Antonioli, ${ }^{8}$ A. Antonov, ${ }^{55}$ B. Antunovic, ${ }^{31}$ M. Arneodo, ${ }^{77}$ V. Aushev, ${ }^{36, a 31}$ O. Bachynska, ${ }^{36}$ S. Backovic, ${ }^{64}$ A. Baghdasaryan, ${ }^{86}$ A. Bamberger, ${ }^{27}$ A.N. Barakbaev, ${ }^{2}$ G. Barbagli, ${ }^{25}$ G. Bari ${ }^{8}$ F. Barreiro, ${ }^{50}$ E. Barrelet, ${ }^{63}$ W. Bartel, ${ }^{31}$ D. Bartsch, ${ }^{10}$ M. Basile ${ }^{9}$ K. Begzsuren, ${ }^{80}$ O. Behnke, ${ }^{31}$ J. Behr, ${ }^{31}$ U. Behrens, ${ }^{31}$ L. Bellagamba,${ }^{8}$ A. Belousov, ${ }^{54}$ A. Bertolin, ${ }^{60}$ S. Bhadra,${ }^{88}$ M. Bindi, ${ }^{9}$ J.C. Bizot, ${ }^{58}$ C. Blohm, ${ }^{31}$ T. Bołd, ${ }^{19}$ E.G. Boos, ${ }^{2}$ M. Borodin, ${ }^{36}$ K. Borras ${ }^{31}$ D. Boscherini, ${ }^{8}$ D. Bot,${ }^{31}$ V. Boudry, ${ }^{62}$ S.K. Boutle,${ }^{42, a 27}$ I. Bozovic-Jelisavcic,${ }^{5}$ J. Bracinik, ${ }^{7}$ G. Brandt,${ }^{31}$ M. Brinkmann, ${ }^{30}$ V. Brisson, ${ }^{58}$ I. Brock, ${ }^{10}$ E. Brownson, ${ }^{49}$ R. Brugnera ${ }^{61}$ N. Brümmer, ${ }^{16}$ D. Bruncko,${ }^{37}$ A. Bruni, ${ }^{8}$ G. Bruni, ${ }^{8}$ B. Brzozowska, ${ }^{83}$ A. Bunyatyan, ${ }^{32,86}$ G. Buschhorn, ${ }^{57}$ P.J. Bussey, ${ }^{29}$ J.M. Butterworth, ${ }^{42}$ B. Bylsma, ${ }^{16}$ L. Bystritskaya, ${ }^{53}$ A. Caldwell, ${ }^{57}$ A.J. Campbell, ${ }^{31}$ K.B. Cantun Avila, ${ }^{47}$ M. Capua, ${ }^{17}$ R. Carlin,${ }^{61}$ C.D. Catterall, ${ }^{88}$ K. Cerny, ${ }^{66}$ V. Cerny, ${ }^{37, a 6}$ S. Chekanov, ${ }^{4}$ V. Chekelian, ${ }^{57}$ A. Cholewa, ${ }^{31}$ J. Chwastowski, ${ }^{18}$ J. Ciborowski, ${ }^{83, a 42}$ R. Ciesielski, ${ }^{31}$ L. Cifarelli, ${ }^{9}$ F. Cindolo, ${ }^{8}$ A. Contin, ${ }^{9}$ J.G. Contreras, ${ }^{47}$ A.M. Cooper-Sarkar, ${ }^{59}$ N. Coppola, ${ }^{31}$ M. Corradi, ${ }^{8}$ F. Corriveau, ${ }^{52}$ M. Costa, ${ }^{76}$ J.A. Coughlan, ${ }^{22}$ G. Cozzika, ${ }^{28}$ J. Cvach, ${ }^{65}$ G. D'Agostini, ${ }^{69}$ J.B. Dainton, ${ }^{41}$ F. Dal Corso, ${ }^{60}$ K. Daum, ${ }^{85, a 2}$ M. Deák, ${ }^{31}$ J. de Favereau, ${ }^{45}$ B. Delcourt, ${ }^{58}$ M. Del Degan,${ }^{90}$ J. del Peso, ${ }^{50}$ J. Delvax, ${ }^{12}$ R.K. Dementiev, ${ }^{56}$ S. De Pasquale, ${ }^{9, a 11}$ M. Derrick ${ }^{4}$ R.C.E. Devenish,${ }^{59}$ E.A. De Wolf, ${ }^{12}$ C. Diaconu,${ }^{51}$ D. Dobur, ${ }^{27}$ V. Dodonov, ${ }^{32}$ B.A. Dolgoshein, ${ }^{55}$ A. Dossanov, ${ }^{57}$ A.T. Doyle, ${ }^{29}$ V. Drugakov, ${ }^{89}$ A. Dubak, ${ }^{64, a 5}$ L.S. Durkin, ${ }^{16}$ S. Dusini, ${ }^{60}$ G. Eckerlin, ${ }^{31}$ V. Efremenko,${ }^{53}$ S. Egli, ${ }^{82}$ Y. Eisenberg, ${ }^{67}$ A. Eliseev, ${ }^{54}$ E. Elsen, ${ }^{31}$ P.F. Ermolov,${ }^{56, \dagger}$ A. Eskreys ${ }^{18}$ A. Falkiewicz, ${ }^{18}$ S. Fang, ${ }^{31}$ L. Favart,${ }^{12}$ S. Fazio, ${ }^{17}$ A. Fedotov,${ }^{53}$ R. Felst, ${ }^{31}$ J. Feltesse, ${ }^{28, a 7}$ J. Ferencei, ${ }^{37}$ J. Ferrando, ${ }^{59}$ M.I. Ferrero, ${ }^{76}$ J. Figiel, ${ }^{18}$ D.-J. Fischer ${ }^{31}$ M. Fleischer, ${ }^{31}$ A. Fomenko, ${ }^{54}$ M. Forrest,${ }^{29}$ B. Foster, ${ }^{59}$ S. Fourletov, ${ }^{78, a 40}$ E. Gabathuler, ${ }^{41}$ A. Galas, ${ }^{18}$ E. Gallo, ${ }^{25}$ A. Garfagnini, ${ }^{61}$ J. Gayler, ${ }^{31}$ A. Geiser, ${ }^{31}$ S. Ghazaryan, ${ }^{86}$ I. Gialas, ${ }^{15, a 27}$ L.K. Gladilin, ${ }^{56}$ D. Gladkov, ${ }^{55}$ C. Glasman, ${ }^{50}$ A. Glazov, ${ }^{31}$ I. Glushkov, ${ }^{89}$ L. Goerlich, ${ }^{18}$ N. Gogitidze, ${ }^{54}$ Yu.A. Golubkov, ${ }^{56}$ P. Göttlicher, ${ }^{31, a 17}$ M. Gouzevitch, ${ }^{31}$ C. Grab, ${ }^{90}$ I. Grabowska-Bołd, ${ }^{19}$ J. Grebenyuk, ${ }^{31}$ T. Greenshaw, ${ }^{41}$ I. Gregor, ${ }^{31}$ B.R. Grell, ${ }^{31}$ G. Grigorescu, ${ }^{3}$ G. Grindhammer, ${ }^{57}$ G. Grzelak, ${ }^{83}$ C. Gwenlan, ${ }^{59, a 33}$ T. Haas, ${ }^{31}$ S. Habib, ${ }^{30}$ D. Haidt, ${ }^{31}$ W. Hain, ${ }^{31}$ R. Hamatsu, ${ }^{75}$ J.C. Hart, ${ }^{22}$ H. Hartmann, ${ }^{10}$ G. Hartner ${ }^{88}$ C. Helebrant,${ }^{31}$ R.C.W. Henderson, ${ }^{40}$ E. Hennekemper, ${ }^{34}$ H. Henschel, ${ }^{89}$ M. Herbst,${ }^{34}$ G. Herrera, ${ }^{48}$ M. Hildebrandt, ${ }^{82}$ E. Hilger, ${ }^{10}$ K.H. Hiller, ${ }^{89}$ D. Hochman, ${ }^{67}$ D. Hoffmann, ${ }^{51}$ U. Holm, ${ }^{30}$ R. Hori,${ }^{74}$ R. Horisberger,${ }^{82}$ K. Horton, ${ }^{59, a 34}$ T. Hreus, ${ }^{12, a 3}$ A. Hüttmann, ${ }^{31}$ G. Iacobucci $^{8}$ Z.A. Ibrahim, ${ }^{38}$ Y. Iga, ${ }^{70}$ 
R. Ingbir, ${ }^{72}$ M. Ishitsuka, ${ }^{73}$ M. Jacquet, ${ }^{58}$ H.-P. Jakob, ${ }^{10}$ X. Janssen, ${ }^{12}$ F. Januschek, ${ }^{31}$ M. Jimenez, ${ }^{50}$ T.W. Jones, ${ }^{42}$ L. Jönsson, ${ }^{46}$ A.W. Jung, ${ }^{34}$ H. Jung, ${ }^{31}$ M. Jüngst, ${ }^{10}$ I. Kadenko, ${ }^{36}$ B. Kahle, ${ }^{31}$ B. Kamaluddin, ${ }^{38}$ S. Kananov, ${ }^{72}$ T. Kanno, ${ }^{73}$ M. Kapichine,${ }^{24}$ U. Karshon, ${ }^{67}$ F. Karstens, ${ }^{27}$ I.I. Katkov, ${ }^{31, a 18}$ J. Katzy, ${ }^{31}$ M. Kaur, ${ }^{14}$ P. Kaur, ${ }^{14, a 13}$ I.R. Kenyon, ${ }^{7}$ A. Keramidas, ${ }^{3}$ L.A. Khein, ${ }^{56}$ C. Kiesling, ${ }^{57}$ J.Y. Kim, ${ }^{39, a 45}$ D. Kisielewska, ${ }^{19}$ S. Kitamura, ${ }^{75, a 37}$ R. Klanner, ${ }^{30}$ M. Klein, ${ }^{41}$ U. Klein, ${ }^{31, a 19}$ C. Kleinwort, ${ }^{31}$ T. Kluge, ${ }^{41}$ A. Knutsson, ${ }^{31}$ E. Koffeman, ${ }^{3}$ R. Kogler, ${ }^{57}$ D. Kollar, ${ }^{57}$ P. Kooijman, ${ }^{3}$ Ie. Korol, ${ }^{36}$ I.A. Korzhavina, ${ }^{56}$ P. Kostka, ${ }^{89}$ A. Kotański, ${ }^{20, a 15}$ U. Kötz ${ }^{31}$ H. Kowalski, ${ }^{31}$ M. Kraemer, ${ }^{30}$ K. Krastev ${ }^{31}$ J. Kretzschmar, ${ }^{41}$ A. Kropivnitskaya, ${ }^{53}$ K. Krüger, ${ }^{34}$ P. Kulinski, ${ }^{83}$ O. Kuprash, ${ }^{36}$ K. Kutak, ${ }^{31}$ M. Kuze, ${ }^{73}$ V.A. Kuzmin, ${ }^{56}$ M.P.J. Landon, ${ }^{43}$ W. Lange, ${ }^{89}$ G. Laštovička-Medin,${ }^{64}$ P. Laycock, ${ }^{41}$ A. Lebedev,${ }^{54}$ A. Lee,${ }^{16}$ G. Leibenguth,${ }^{90}$ V. Lendermann, ${ }^{34}$ B.B. Levchenko, ${ }^{56, a 32}$ S. Levonian, ${ }^{31}$ A. Levy, ${ }^{72}$ G. Li, ${ }^{58}$ V. Libov, ${ }^{36}$ S. Limentani, ${ }^{61}$ T.Y. Ling, ${ }^{16}$ K. Lipka, ${ }^{31}$ A. Liptaj, ${ }^{57}$ M. Lisovyi,${ }^{31}$ B. List,${ }^{30}$ J. List,${ }^{31}$ E. Lobodzinska, ${ }^{31}$ W. Lohmann, ${ }^{89}$ B. Löhr,${ }^{31}$ E. Lohrmann, ${ }^{30}$ J.H. Loizides, ${ }^{42}$ N. Loktionova, ${ }^{54}$ K.R. Long, ${ }^{44}$ A. Longhin, ${ }^{60}$ D. Lontkovskyi,${ }^{36}$ R. Lopez-Fernandez, ${ }^{48}$ V. Lubimov, ${ }^{53}$ J. Lukasik, ${ }^{19, a 14}$ O.Yu. Lukina, ${ }^{56}$ P. Łużniak, ${ }^{83, a 43}$ J. Maeda, ${ }^{73}$ S. Magill, ${ }^{4}$ A. Makankine, ${ }^{24}$ I. Makarenko, ${ }^{36}$ E. Malinovski, ${ }^{54}$ J. Malka, ${ }^{83, a 43}$ R. Mankel, ${ }^{31, a 20}$ P. Marage, ${ }^{12}$ A. Margotti, ${ }^{8}$ G. Marini, ${ }^{69}$ Ll. Marti, ${ }^{31}$ J.F. Martin, ${ }^{78}$ H.-U. Martyn, ${ }^{1}$ A. Mastroberardino, ${ }^{17}$ T. Matsumoto, ${ }^{79, a 28}$ M.C.K. Mattingly, ${ }^{6}$ S.J. Maxfield, ${ }^{41}$ A. Mehta, ${ }^{41}$ I.-A. Melzer-Pellmann, ${ }^{31}$ A.B. Meyer, ${ }^{31}$ H. Meyer,${ }^{31}$ H. Meyer, ${ }^{85}$ J. Meyer, ${ }^{31}$ S. Miglioranzi, ${ }^{31, a 21}$ S. Mikocki, ${ }^{18}$ I. Milcewicz-Mika, ${ }^{18}$ F. Mohamad Idris, ${ }^{38}$ V. Monaco ${ }^{76}$ A. Montanari ${ }^{31}$ F. Moreau, ${ }^{62}$ A. Morozov, ${ }^{24}$ J.D. Morris, ${ }^{11, a 12}$ J.V. Morris, ${ }^{22}$ M.U. Mozer, ${ }^{12}$ M. Mudrinic ${ }^{5}$ K. Müller, ${ }^{91}$ P. Murín, ${ }^{37, a 3}$ B. Musgrave, ${ }^{4}$ K. Nagano, ${ }^{79}$ T. Namsoo, ${ }^{31}$ R. Nania, ${ }^{8}$ Th. Naumann, ${ }^{89}$ P.R. Newman, ${ }^{7}$ D. Nicholass, ${ }^{4, a 10}$ C. Niebuhr, ${ }^{31}$ A. Nigro, ${ }^{69}$ A. Nikiforov, ${ }^{31}$ D. Nikitin, ${ }^{24}$ Y. Ning, ${ }^{35}$ U. Noor,${ }^{88}$ D. Notz,${ }^{31}$ G. Nowak, ${ }^{18}$ K. Nowak, ${ }^{91}$ R.J. Nowak, ${ }^{83}$ M. Nozicka, ${ }^{31}$ A.E. Nuncio-Quiroz, ${ }^{10}$ B.Y. Oh, ${ }^{81}$ N. Okazaki, ${ }^{74}$ K. Oliver, ${ }^{59}$ B. Olivier, ${ }^{57}$ K. Olkiewicz, ${ }^{18}$ J.E. Olsson, ${ }^{31}$ Yu. Onishchuk, ${ }^{36}$ S. Osman, ${ }^{46}$ O. Ota, ${ }^{75, a 38}$ D. Ozerov ${ }^{53}$ V. Palichik, ${ }^{24}$ I. Panagoulias, ${ }^{31, a 1, b 13}$ M. Pandurovic, ${ }^{5}$ Th. Papadopoulou, ${ }^{31, a 1, b 13}$ K. Papageorgiu, ${ }^{15}$ A. Parenti, ${ }^{31}$ C. Pascaud, ${ }^{58}$ G.D. Patel,${ }^{41}$ E. Paul, ${ }^{10}$ J.M. Pawlak, ${ }^{83}$ B. Pawlik, ${ }^{18}$ O. Pejchal, ${ }^{66}$ P.G. Pelfer, ${ }^{26}$ A. Pellegrino,${ }^{3}$ E. Perez, ${ }^{28, a 4}$ W. Perlanski, ${ }^{83, a 43}$ H. Perrey, ${ }^{30}$ A. Petrukhin, ${ }^{53}$ I. Picuric, ${ }^{64}$ S. Piec, ${ }^{89}$ K. Piotrzkowski, ${ }^{45}$ D. Pitzl, ${ }^{31}$ R. Plačakytė, ${ }^{31}$ P. Plucinski, ${ }^{84, a 44}$ B. Pokorny,${ }^{30}$ N.S. Pokrovskiy, ${ }^{2}$ R. Polifka, ${ }^{66}$ A. Polini, ${ }^{8}$ B. Povh, ${ }^{32}$ A.S. Proskuryakov, ${ }^{56}$ M. Przybycień, ${ }^{19}$ V. Radescu, ${ }^{31}$ A.J. Rahmat, ${ }^{41}$ N. Raicevic, ${ }^{64}$ A. Raspiareza, ${ }^{57}$ A. Raval, ${ }^{81}$ T. Ravdandorj, ${ }^{80}$ D.D. Reeder ${ }^{49}$ P. Reimer, ${ }^{65}$ B. Reisert,${ }^{57}$ Z. Ren, ${ }^{35}$ J. Repond, ${ }^{4}$ Y.D. Ri, ${ }^{75, a 39}$ E. Rizvi, ${ }^{43}$ A. Robertson, ${ }^{59}$ P. Robmann, ${ }^{91}$ B. Roland, ${ }^{12}$ P. Roloff, ${ }^{31}$ E. Ron, ${ }^{50}$ R. Roosen, ${ }^{12}$ A. Rostovtsev, ${ }^{53}$ M. Rotaru, ${ }^{13}$ I. Rubinsky, ${ }^{31}$ J.E. Ruiz Tabasco, ${ }^{47}$ Z. Rurikova, ${ }^{31}$ S. Rusakov, ${ }^{54}$ M. Ruspa, ${ }^{77}$ R. Sacchi, ${ }^{76}$ 
D. Sálek, ${ }^{66}$ A. Salii, ${ }^{36}$ U. Samson, ${ }^{10}$ D.P.C. Sankey,${ }^{22}$ G. Sartorelli, ${ }^{9}$ M. Sauter,${ }^{90}$ E. Sauvan, ${ }^{51}$ A.A. Savin, ${ }^{49}$ D.H. Saxon, ${ }^{29}$ M. Schioppa, ${ }^{17}$ S. Schlenstedt,${ }^{89}$ P. Schleper, ${ }^{30}$ W.B. Schmidke, ${ }^{57}$ S. Schmitt, ${ }^{31}$ U. Schneekloth, ${ }^{31}$ L. Schoeffel, ${ }^{28}$ V. Schönberg ${ }^{10}$ A. Schöning, ${ }^{33}$ T. Schörner-Sadenius, ${ }^{30}$ H.-C. Schultz-Coulon, ${ }^{34}$ J. Schwartz, ${ }^{52}$ F. Sciulli, ${ }^{35}$ F. Sefkow, ${ }^{31}$ R.N. Shaw-West, ${ }^{7}$ L.M. Shcheglova, ${ }^{56}$ R. Shehzadi, ${ }^{10}$ S. Shimizu, ${ }^{74, a 21}$ L.N. Shtarkov, ${ }^{54}$ S. Shushkevich, ${ }^{57}$ I. Singh, ${ }^{14, a 13}$ I.O. Skillicorn, ${ }^{29}$ T. Sloan, ${ }^{40}$ W. Słomiński, ${ }^{20, a 16}$ I. Smiljanic ${ }^{5}$ W.H. Smith, ${ }^{49}$ V. Sola, ${ }^{76}$ A. Solano, ${ }^{76}$ Y. Soloviev, ${ }^{54}$ D. Son, ${ }^{21}$ P. Sopicki, ${ }^{18}$ Iu. Sorokin,,${ }^{36}$ V. Sosnovtsev, ${ }^{55}$ D. South, ${ }^{23}$ V. Spaskov,${ }^{24}$ A. Specka, ${ }^{62}$ A. Spiridonov, ${ }^{31, a 22}$ H. Stadie,${ }^{30}$ L. Stanco,${ }^{60}$ Z. Staykova, ${ }^{31}$ M. Steder,${ }^{31}$ B. Stella, ${ }^{68}$ A. Stern,${ }^{72}$ T.P. Stewart, ${ }^{78}$ A. Stifutkin, ${ }^{55}$ G. Stoicea, ${ }^{13}$ P. Stopa, ${ }^{18}$ U. Straumann, ${ }^{91}$ S. Suchkov, ${ }^{55}$ D. Sunar, ${ }^{12}$ G. Susinno, ${ }^{17}$ L. Suszycki, ${ }^{19}$ T. Sykora, ${ }^{12}$ J. Sztuk, ${ }^{30}$ D. Szuba ${ }^{31, a 23}$ J. Szuba, ${ }^{31, a 24}$ A.D. Tapper, ${ }^{44}$ E. Tassi,${ }^{17, a 41}$ V. Tchoulakov, ${ }^{24}$ J. Terrón, ${ }^{50}$ T. Theedt,${ }^{31}$ G. Thompson, ${ }^{43}$ P.D. Thompson, ${ }^{7}$ H. Tiecke,${ }^{3}$ K. Tokushuku, ${ }^{79, a 29}$ T. Toll, ${ }^{30}$ F. Tomasz,${ }^{37}$ J. Tomaszewska, ${ }^{31, a 25}$ T.H. Tran, ${ }^{58}$ D. Traynor, ${ }^{43}$ T.N. Trinh, ${ }^{51}$ P. Truöl, ${ }^{91}$ I. Tsakov, ${ }^{71}$ B. Tseepeldorj, ${ }^{80, a 9}$ T. Tsurugai, ${ }^{87}$ M. Turcato, ${ }^{30}$ J. Turnau, ${ }^{18}$ T. Tymieniecka, ${ }^{84, a 47}$ K. Urban, ${ }^{34}$ C. Uribe-Estrada, ${ }^{50}$ A. Valkárová, ${ }^{66}$ C. Vallée, ${ }^{51}$ P. Van Mechelen, ${ }^{12}$ A. Vargas Trevino, ${ }^{31}$ Y. Vazdik, ${ }^{54}$ M. Vázquez, ${ }^{3, a 21}$ A. Verbytskyi, ${ }^{36}$ V. Viazlo, ${ }^{36}$ S. Vinokurova, ${ }^{31}$ N.N. Vlasov,${ }^{27, a 26}$ V. Volchinski, ${ }^{86}$ O. Volynets, ${ }^{36}$ M. von den Driesch, ${ }^{31}$ R. Walczak, ${ }^{59}$ W.A.T. Wan Abdullah, ${ }^{38}$ D. Wegener, ${ }^{23}$ J.J. Whitmore, ${ }^{81, a 35}$ J. Whyte, ${ }^{88}$ L. Wiggers, ${ }^{3}$ M. Wing, ${ }^{42, a 46}$ Ch. Wissing, ${ }^{31}$ M. Wlasenko, ${ }^{10}$ G. Wolf, ${ }^{31}$ H. Wolfe, ${ }^{49}$ K. Wrona, ${ }^{31}$ E. Wünsch, ${ }^{31}$ A.G. Yagües-Molina, ${ }^{31}$ S. Yamada, $^{79}$ Y. Yamazaki, ${ }^{79, a 30}$ R. ${ }^{\text {Yoshida, }}{ }^{4}$ C. Youngman, ${ }^{31}$ J. Žáček, ${ }^{66}$ J. Zálešák, ${ }^{65}$ A.F. Żarnecki, ${ }^{83}$ L. Zawiejski, ${ }^{18}$ O. Zenaiev, ${ }^{36}$ W. Zeuner, ${ }^{31, a 20}$ Z. Zhang, ${ }^{58}$ B.O. Zhautykov, ${ }^{2}$ A. Zhokin, ${ }^{53}$ C. Zhou, ${ }^{52}$ A. Zichichi, ${ }^{9}$ T. Zimmermann, ${ }^{90}$ H. Zohrabyan, ${ }^{86}$ M. Zolko, ${ }^{36}$ F. Zomer, ${ }^{58}$ D.S. Zotkin ${ }^{56}$ and R. Zus ${ }^{13}$

${ }^{1}$ I. Physikalisches Institut der RWTH, Aachen, Germany

${ }^{2}$ Institute of Physics and Technology of Ministry of Education and Science of Kazakhstan, Almaty, Kazakhstan

${ }^{3}$ NIKHEF and University of Amsterdam, Amsterdam, Netherlands ${ }^{b 20}$

${ }^{4}$ Argonne National Laboratory, Argonne, Illinois 60439-4815, U.S.A. ${ }^{625}$

${ }^{5}$ Vinca Institute of Nuclear Sciences, Belgrade, Serbia

${ }^{6}$ Andrews University, Berrien Springs, Michigan 49104-0380, U.S.A.

7 School of Physics and Astronomy, University of Birmingham, Birmingham, U.K. ${ }^{b 24}$

${ }^{8}$ INFN Bologna, Bologna, Italy ${ }^{b 17}$

${ }^{9}$ University and INFN Bologna, Bologna, Italy ${ }^{b 17}$

${ }^{10}$ Physikalisches Institut der Universität Bonn, Bonn, Germany ${ }^{b 2}$

${ }^{11}$ H.H. Wills Physics Laboratory, University of Bristol, Bristol, U.K. ${ }^{624}$

12 Inter-University Institute for High Energies ULB-VUB,

Brussels, Universiteit Antwerpen, Antwerpen, Belgium ${ }^{63}$

${ }^{13}$ National Institute for Physics and Nuclear Engineering (NIPNE), Bucharest, Romania 
14 Panjab University, Department of Physics, Chandigarh, India

15 Department of Engineering in Management and Finance, Univ. of the Aegean, Chios, Greece

16 Physics Department, Ohio State University, Columbus, Ohio 43210, U.S.A. ${ }^{b 25}$

17 Calabria University, Physics Department and INFN, Cosenza, Italy ${ }^{b 17}$

18 The Henryk Niewodniczanski Institute of Nuclear Physics,

Polish Academy of Sciences, Cracow, Poland ${ }^{b 4, b 5}$

19 Faculty of Physics and Applied Computer Science, AGH-University of Science and Technology, Cracow, Poland ${ }^{b 27}$

20 Department of Physics, Jagellonian University, Cracow, Poland

${ }^{21}$ Kyungpook National University, Center for High Energy Physics, Daegu, South Korea ${ }^{b 19}$

${ }^{22}$ Rutherford Appleton Laboratory, Chilton, Didcot, U.K. ${ }^{b 24}$

23 Institut für Physik, TU Dortmund, Dortmund, Germany ${ }^{b 1}$

24 Joint Institute for Nuclear Research, Dubna, Russia

${ }^{25}$ INFN Florence, Florence, Italy ${ }^{b 17}$

26 University and INFN Florence, Florence, Italy ${ }^{b 17}$

27 Fakultät für Physik der Universität Freiburg i.Br., Freiburg i.Br., Germany ${ }^{b 2}$

28 CEA, DSM/Irfu, CE-Saclay, Gif-sur-Yvette, France

29 Department of Physics and Astronomy, University of Glasgow, Glasgow, U.K. ${ }^{b 24}$

30 Institut für Experimentalphysik, Universität Hamburg, Hamburg, Germany ${ }^{b 1, b 2}$

31 Deutsches Elektronen-Synchrotron DESY, Hamburg, Germany

32 Max-Planck-Institut für Kernphysik, Heidelberg, Germany

33 Physikalisches Institut, Universität Heidelberg, Heidelberg, Germany ${ }^{b 1}$

${ }^{34}$ Kirchhoff-Institut für Physik, Universität Heidelberg, Heidelberg, Germany ${ }^{b 1}$

35 Nevis Laboratories, Columbia University, Irvington on Hudson, New York 10027, U.S.A. ${ }^{b 26}$

36 Institute for Nuclear Research, National Academy of Sciences and Kiev National University, Kiev, Ukraine

37 Institute of Experimental Physics, Slovak Academy of Sciences, Košice, Slovak Republic ${ }^{b 7}$

38 Jabatan Fizik, Universiti Malaya, 50603 Kuala Lumpur, Malaysiab29

39 Chonnam National University, Kwangju, South Korea

40 Department of Physics, University of Lancaster, Lancaster, U.K. ${ }^{b 24}$

41 Department of Physics, University of Liverpool, Liverpool, U.K. ${ }^{b 24}$

42 Physics and Astronomy Department, University College London, London, U.K. ${ }^{b 24}$

43 Queen Mary and Westfield College, London, U.K. ${ }^{b 24}$

${ }^{44}$ Imperial College London, High Energy Nuclear Physics Group, London, U.K. ${ }^{b 24}$

45 Institut de Physique Nucléaire, Université Catholique de Louvain,

Louvain-la-Neuve, Belgium ${ }^{\text {b28 }}$

46 Physics Department, University of Lund, Lund, Sweden ${ }^{b 8}$

47 Departamento de Fisica Aplicada, CINVESTAV, Mérida Yucatán, Mexico ${ }^{b 11}$

48 Departamento de Fisica, CINVESTAV, México City, Mexico ${ }^{b 11}$

49 Department of Physics, University of Wisconsin, Madison, Wisconsin 53706, U.S.A. ${ }^{\text {b25 }}$

50 Departamento de Física Teórica, Universidad Autónoma de Madrid, Madrid, Spain ${ }^{b 23}$

51 CPPM, CNRS/IN2P3 - Univ. Mediterranee, Marseille, France

52 Department of Physics, McGill University, Montréal, Québec, Canada H3A 2T8 14 
53 Institute for Theoretical and Experimental Physics, Moscow, Russia ${ }^{b 12}$

${ }^{54}$ Lebedev Physical Institute, Moscow, Russia ${ }^{b 6}$

55 Moscow Engineering Physics Institute, Moscow, Russiab ${ }^{b 21}$

56 Moscow State University, Institute of Nuclear Physics, Moscow, Russia ${ }^{b 22}$

57 Max-Planck-Institut für Physik, München, Germany

58 LAL, Univ Paris-Sud, CNRS/IN2P3, Orsay, France

59 Department of Physics, University of Oxford, Oxford, U.K. ${ }^{b 24}$

60 INFN Padova, Padova, Italy ${ }^{b 17}$

61 Dipartimento di Fisica dell'Università and INFN, Padova, Italy ${ }^{b 17}$

62 LLR, Ecole Polytechnique, CNRS/IN2P3, Palaiseau, France

${ }^{63}$ LPNHE, Universités Paris VI and VII, CNRS/IN2P3, Paris, France

${ }^{64}$ Faculty of Science, University of Montenegro, Podgorica, Montenegro ${ }^{b 6}$

65 Institute of Physics, Academy of Sciences of the Czech Republic, Praha, Czech Republic ${ }^{b 9}$

66 Faculty of Mathematics and Physics, Charles University, Praha, Czech Republic ${ }^{b 9}$

67 Department of Particle Physics, Weizmann Institute, Rehovot, Israel ${ }^{b 15}$

68 Dipartimento di Fisica Università di Roma Tre and INFN Roma 3, Roma, Italy

69 Dipartimento di Fisica, Università 'La Sapienza' and INFN, Rome, Italy ${ }^{b 17}$

70 Polytechnic University, Sagamihara, Japan ${ }^{b 18}$

${ }^{71}$ Institute for Nuclear Research and Nuclear Energy, Sofia, Bulgaria ${ }^{b 6}$

72 Raymond and Beverly Sackler Faculty of Exact Sciences, School of Physics,

Tel Aviv University, Tel Aviv, Israel ${ }^{b 16}$

73 Department of Physics, Tokyo Institute of Technology, Tokyo, Japan ${ }^{b 18}$

74 Department of Physics, University of Tokyo, Tokyo, Japan ${ }^{b 18}$

75 Tokyo Metropolitan University, Department of Physics, Tokyo, Japan ${ }^{b 18}$

76 Università di Torino and INFN, Torino, Italy ${ }^{b 17}$

77 Università del Piemonte Orientale, Novara, and INFN, Torino, Italy ${ }^{b 17}$

78 Department of Physics, University of Toronto, Toronto, Ontario, Canada M5S $1 A$ rp14

79 Institute of Particle and Nuclear Studies, KEK, Tsukuba, Japan ${ }^{b 18}$

80 Institute of Physics and Technology of the Mongolian Academy of Sciences,

Ulaanbaatar, Mongolia

81 Department of Physics, Pennsylvania State University,

University Park, Pennsylvania 16802, U.S.A. ${ }^{b 26}$

82 Paul Scherrer Institut, Villigen, Switzerland

83 Warsaw University, Institute of Experimental Physics, Warsaw, Poland

84 Institute for Nuclear Studies, Warsaw, Poland

${ }^{85}$ Fachbereich C, Universität Wuppertal, Wuppertal, Germany

86 Yerevan Physics Institute, Yerevan, Armenia

${ }^{87}$ Meiji Gakuin University, Faculty of General Education, Yokohama, Japan ${ }^{b 18}$

${ }^{8}$ Department of Physics, York University, Ontario, Canada M3J1P3 $3^{b 14}$

89 Deutsches Elektronen-Synchrotron DESY, Zeuthen, Germany

90 Institut für Teilchenphysik, ETH, Zürich, Switzerland $d^{b 10}$

91 Physik-Institut der Universität Zürich, Zürich, Switzerland ${ }^{b 10}$ 
$a_{1}$ Also at Physics Department, National Technical University, Zografou Campus, GR-15773 Athens, Greece

${ }^{a 2}$ Also at Rechenzentrum, Universität Wuppertal, Wuppertal, Germany

${ }^{a 3}$ Also at University of P.J. Šafárik, Košice, Slovak Republic

${ }^{a}$ Also at CERN, Geneva, Switzerland

${ }^{a 5}$ Also at Max-Planck-Institut für Physik, München, Germany

${ }^{a 6}$ Also at Comenius University, Bratislava, Slovak Republic

${ }^{a 7}$ Also at DESY and University Hamburg, Helmholtz Humboldt Research Award

${ }^{a 8}$ Also at Faculty of Physics, University of Bucharest, Bucharest, Romania

${ }^{a 9}$ Also at Ulaanbaatar University, Ulaanbaatar, Mongolia

${ }^{a 10}$ Also affiliated with University College London, U.K.

${ }^{a 11}$ Now at University of Salerno, Italy

$a_{12}$ Now at Queen Mary University of London, U.K.

${ }^{a 13}$ Also working at Max Planck Institute, Munich, Germany

${ }^{a 14}$ Now at Institute of Aviation, Warsaw, Poland

${ }^{a 15}$ Supported by the research grant No. 1 P03B 04529 (2005-2008)

${ }_{a 16}$ This work was supported in part by the Marie Curie Actions Transfer of Knowledge project COCOS (contract MTKD-CT-2004-517186)

${ }^{a 17}$ Now at DESY group FEB, Hamburg, Germany

${ }^{a 18}$ Also at Moscow State University, Russia

${ }^{a 19}$ Now at University of Liverpool, U.K.

${ }^{a 20}$ On leave of absence at CERN, Geneva, Switzerland

${ }^{a 21}$ Now at CERN, Geneva, Switzerland

${ }^{a 22}$ Also at Institut of Theoretical and Experimental Physics, Moscow, Russia

a23 Also at INP, Cracow, Poland

${ }^{a 24}$ Also at FPACS, AGH-UST, Cracow, Poland

${ }^{a 25}$ Partially supported by Warsaw University, Poland

${ }^{a 26}$ Partially supported by Moscow State University, Russia

${ }^{a 27}$ Also affiliated with DESY, Germany

${ }^{a} 28$ Now at Japan Synchrotron Radiation Research Institute (JASRI), Hyogo, Japan

${ }^{a 29}$ Also at University of Tokyo, Japan

${ }^{a 30}$ Now at Kobe University, Japan

${ }^{a 31}$ Supported by DESY, Germany

${ }_{a 32}$ Partially supported by Russian Foundation for Basic Research grant No. 05-02-39028-NSFC-a

a33 STFC Advanced Fellow

${ }^{a 34}$ Nee Korcsak-Gorzo

a35 This material was based on work supported by the National Science Foundation, while working at the Foundation.

${ }^{a 36}$ Also at Max Planck Institute, Munich, Germany, Alexander von Humboldt Research Award

${ }^{a 37}$ Now at Nihon Institute of Medical Science, Japan

${ }^{a} 38$ Now at SunMelx Co. Ltd., Tokyo, Japan

a39 Now at Osaka University, Osaka, Japan

${ }^{a 40}$ Now at University of Bonn, Germany

${ }^{a 41}$ also Senior Alexander von Humboldt Research Fellow at Hamburg University

${ }^{a 42}$ Also at Łódź University, Poland

${ }^{a 43}$ Member of Łódź University, Poland

${ }^{a 44}$ Now at Lund University, Lund, Sweden

${ }^{a 45}$ Supported by Chonnam National University, South Korea, in 2009 
${ }^{a 46}$ Also at Hamburg University, Inst. of Exp. Physics, Alexander von Humboldt Research Award and partially supported by DESY, Hamburg, Germany

${ }^{a 47}$ Also at University of Podlasie, Siedlce, Poland

${ }^{b 1}$ Supported by the German Federal Ministry for Education and Research (BMBF), under contract numbers 05H09GUF, 05H09VHC, 05H09VHF and 05H16PEA

${ }^{b 2}$ Supported by the German Federal Ministry for Education and Research (BMBF), under contract numbers 05 HZ6PDA, 05 HZ6GUA, 05 HZ6VFA and 05 HZ4KHA

${ }^{b 3}$ Supported by FNRS-FWO-Vlaanderen, IISN-IIKW and IWT and by Interuniversity Attraction Poles Programme, Belgian Science Policy

${ }^{b 4}$ Supported by the Polish State Committee for Scientific Research, project No. DESY/256/2006 - 154/DES/2006/03

b5 Partially Supported by Polish Ministry of Science and Higher Education, grant PBS/DESY/70/2006

${ }^{b 6}$ Supported by the Deutsche Forschungsgemeinschaft

${ }^{b 7}$ Supported by VEGA SR grant no. 2/7062/ 27

${ }^{b 8}$ Supported by the Swedish Natural Science Research Council

${ }^{b 9}$ Supported by the Ministry of Education of the Czech Republic under the projects LC527, INGO1P05LA259 and MSM0021620859

${ }^{b 10}$ Supported by the Swiss National Science Foundation

${ }^{b 11}$ Supported by CONACYT, México, grant 48778-F

${ }^{b 12}$ Russian Foundation for Basic Research (RFBR), grant no 1329.2008.2

${ }^{b 13}$ This project is co-funded by the European Social Fund (75\% and National Resources (25\%) (EPEAEK II) - PYTHAGORAS II

${ }^{b 14}$ Supported by the Natural Sciences and Engineering Research Council of Canada (NSERC)

${ }^{b 15}$ Supported in part by the MINERVA Gesellschaft für Forschung GmbH, the Israel Science Foundation (grant No. 293/02-11.2) and the US-Israel Binational Science Foundation

${ }^{b 16}$ Supported by the Israel Science Foundation

${ }^{b 17}$ Supported by the Italian National Institute for Nuclear Physics (INFN)

${ }^{b 18}$ Supported by the Japanese Ministry of Education, Culture, Sports, Science and Technology (MEXT) and its grants for Scientific Research

${ }^{b 19}$ Supported by the Korean Ministry of Education and Korea Science and Engineering Foundation

${ }^{b 20}$ Supported by the Netherlands Foundation for Research on Matter (FOM)

${ }^{b 21}$ Partially supported by the German Federal Ministry for Education and Research (BMBF)

${ }^{b 22}$ Supported by RF Presidential grant N 1456.2008.2 for the leading scientific schools and by the

Russian Ministry of Education and Science through its grant for Scientific Research on High Energy Physics

${ }^{b 23}$ Supported by the Spanish Ministry of Education and Science through funds provided by CICYT

${ }^{b 24}$ Supported by the UK Science and Technology Facilities Council

${ }^{b 25}$ Supported by the US Department of Energy

${ }^{b 26}$ Supported by the US National Science Foundation. Any opinion, findings and conclusions or recommendations expressed in this material are those of the authors and do not necessarily reflect the views of the National Science Foundation.

${ }^{b 27}$ Supported by the Polish Ministry of Science and Higher Education as a scientific project (20092010)

${ }^{b 28}$ Supported by FNRS and its associated funds (IISN and FRIA) and by an Inter-University Attraction Poles Programme subsidised by the Belgian Federal Science Policy Office

${ }^{b 29}$ Supported by an FRGS grant from the Malaysian government

$\dagger$ deceased 


\section{Contents}

The H1 and ZEUS collaborations

1 Introduction $\quad 1$

2 Standard model processes 2

3 Experimental method 2

4 Results 5

$\begin{array}{llr}5 & \text { Conclusions } & 6\end{array}$

\section{Introduction}

In the Standard Model (SM) events containing an isolated electron ${ }^{1}$ or muon of high transverse momentum, $P_{T}$, in coincidence with large missing transverse momentum, $P_{T}^{\text {miss }}$, arise from the production of single $W$ bosons with subsequent decay to leptons. Events of this topology have been observed at the electron-proton collider HERA [1-4]. An excess of events containing in addition a hadronic final state of high transverse momentum, $P_{T}^{X}$, was previously reported by the $\mathrm{H} 1$ collaboration in $105 \mathrm{pb}^{-1}$ of $e^{+} p$ data [3]. Both the $\mathrm{H} 1$ and ZEUS collaborations have recently performed a search for such events using their complete $e^{ \pm} p$ high energy data, corresponding to an integrated luminosity of approximately $0.5 \mathrm{fb}^{-1}$ per experiment $[5,6]$. The event yields are found to be in good overall agreement with the $\mathrm{SM}$ and a measurement of single $W$ production is performed by both collaborations. An excess of events is however still seen by $\mathrm{H} 1$ at high $P_{T}^{X}>25 \mathrm{GeV}$ in the $e^{+} p$ data sample, where 17 events are observed compared to a SM prediction of $8.0 \pm 1.3$ [6].

This paper presents a combined analysis of the H1 and ZEUS data, performed in a common phase space. The analysis makes use of the full data samples available to both experiments allowing a more accurate measurement, as well as a more stringent examination of the high $P_{T}^{X}$ region. Total event yields and kinematic distributions of events containing an isolated electron or muon of high transverse momentum and missing transverse momentum are compared to the SM. In addition, total and differential cross sections for single $W$ production are measured.

The analysed data were collected between 1994 and 2007 at HERA using the H1 and ZEUS detectors. The electron and proton beam energies were $27.6 \mathrm{GeV}$ and $820 \mathrm{GeV}$ or $920 \mathrm{GeV}$ respectively, corresponding to centre-of-mass energies, $\sqrt{s}$, of $301 \mathrm{GeV}$ or $319 \mathrm{GeV}$. The data correspond to an integrated luminosity of $0.98 \mathrm{fb}^{-1}$ comprising $0.39 \mathrm{fb}^{-1}$ of $e^{-} p$

\footnotetext{
${ }^{1}$ Here and in the following, the term "electron" denotes generically both the electron and the positron.
} 
collisions and $0.59 \mathrm{fb}^{-1}$ of $e^{+} p$ collisions, with $9 \%$ of the total integrated luminosity collected at $\sqrt{s}=301 \mathrm{GeV}$. Data collected from 2003 onwards were taken with a longitudinally polarised lepton beam, with polarisation typically at a level of $35 \%$. The residual polarisation of the combined left-handed and right-handed data periods is less than $3 \%$ for both experiments.

\section{$2 \quad$ Standard model processes}

In this analysis, SM processes are considered signal if they produce events containing a high $P_{T}$ isolated charged lepton and at least one high $P_{T}$ neutrino, which escapes detection and leads to $P_{T}^{\text {miss }}$ in the final state. The production of single $W$ bosons with subsequent decay to an electron or a muon, which includes a contribution from leptonic tau-decay, is the main signal contribution to the SM expectation. The EPVEC [11] Monte Carlo (MC) event generator is used to calculate the single $W$ production cross section. The $e p \rightarrow e W X$ events from EPVEC are weighted by a factor dependent on the transverse momentum and rapidity of the $W$, such that the resulting cross section corresponds to a calculation including Quantum Chromodynamics (QCD) corrections at next-to-leading order (NLO) [12-14]. The estimated uncertainty on this calculation is $15 \%$, which arises from the uncertainties in the parton densities and the scale at which the calculation is performed. The contribution of $e p \rightarrow \nu_{e} W X$ events to the total single $W$ production cross section is approximately $7 \%$. The process $e p \rightarrow e Z(\rightarrow \nu \bar{\nu}) X$ also produces high $P_{T}$ isolated electrons and large $P_{T}^{\text {miss }}$ in the final state. The visible cross section for this process as calculated by EPVEC is less than $3 \%$ of the predicted single $W$ production cross section and is neglected in the ZEUS part of the analysis.

All other SM processes are defined as background and contribute to the selected sample mainly through misidentification or mismeasurement. Neutral current (NC) deep inelastic scattering (DIS) events $(e p \rightarrow e X)$, in which genuine isolated high $P_{T}$ electrons are produced, form a significant background in the electron channel when fake $P_{T}^{\text {miss }}$ arises from mismeasurement. Charged current (CC) DIS events $\left(e p \rightarrow \nu_{e} X\right)$, in which there is real $P_{T}^{\text {miss }}$ due to the escaping neutrino, contribute to the background when fake isolated electrons or muons are observed. Lepton pair production $\left(e p \rightarrow e \ell^{+} \ell^{-} X\right)$ contributes to the background via events where one lepton escapes detection and/or measurement errors cause apparent missing momentum. A small contribution to the background in the electron channel arises from QED Compton (QEDC) events $(e p \rightarrow e \gamma X)$ when mismeasurement leads to apparent missing momentum. The background contribution to the analysis from photoproduction is negligible.

\section{Experimental method}

The H1 and ZEUS detectors are general purpose instruments which consist of tracking systems surrounded by electromagnetic and hadronic calorimeters and muon detectors, ensuring close to $4 \pi$ coverage of the $e^{ \pm} p$ interaction point. The origin of the coordinate system is the nominal $e^{ \pm} p$ interaction point, with the direction of the proton beam defining 
the positive $z$-axis (forward region). The $x-y$ plane is called the transverse plane and $\phi$ is the azimuthal angle. The pseudorapidity $\eta$ is defined as $\eta=-\ln \tan (\theta / 2)$, where $\theta$ is the polar angle. Detailed descriptions of the detectors can be found elsewhere [7-10].

The event selection for isolated electrons or muons and missing transverse momentum is based on those used by the H1 [6] and ZEUS [5] experiments. For the combined analysis, a common phase space is chosen in a region where both detectors have a high and well understood acceptance. The event selection for the electron and muon channels is summarised in table 1, and uses the variables described below.

Leptons are identified according to the selection criteria employed by the individual experiments $[5,6]$. Electron candidates are identified as compact and isolated energy deposits in the electromagnetic calorimeters associated to a track in the inner tracking system. Muon candidates are identified as tracks from the inner tracking system associated with track segments reconstructed in muon chambers or energy deposits in the calorimeters compatible with a minimum ionising particle. Lepton candidates are required to lie within the polar angle range $15^{\circ}<\theta_{\ell}<120^{\circ}$ and to have transverse momentum, $P_{T}^{\ell}$, greater than $10 \mathrm{GeV}$. The lepton is required to be isolated with respect to jets and other tracks in the event. Jets are reconstructed from particles in the event not previously identified as isolated leptons using an inclusive $k_{T}$ algorithm $[15,16]$. The isolation of the lepton is quantified using the distances in $\eta$ - $\phi$ space to the nearest jet $D(\ell ;$ jet $)>1.0$ and nearest track $D(\ell$; track $)>0.5$. To ensure that the two channels are exclusive, electron channel events must contain no isolated muons.

The selected events should contain a large transverse momentum imbalance $P_{T}^{\text {miss }}>$ $12 \mathrm{GeV}$. To ensure a high trigger efficiency, the transverse momentum measured in the calorimeter, $P_{T}^{\text {calo }}$, is also required to be greater than $12 \mathrm{GeV}$. As muons deposit little energy in the calorimeter, $P_{T}^{\text {calo }}$ is similar to $P_{T}^{X}$ in the muon channel and therefore the $P_{T}^{\text {calo }}$ requirement effectively acts as a cut on $P_{T}^{X}$. For this reason, the muon channel is restricted to the region $P_{T}^{X}>12 \mathrm{GeV}$.

In order to reduce the remaining SM background, a series of further cuts are applied as described in table 1. A measure of the azimuthal balance of the event, $V_{\text {ap }} / V_{\mathrm{p}}$, is defined as the ratio of the anti-parallel to parallel momentum components of all measured calorimetric clusters with respect to the direction of the total calorimetric transverse momentum [17]. The difference in azimuthal angle between the lepton and the direction of the hadronic system, $\Delta \phi_{\ell-X}$, is used to reject SM background with back-to-back topologies $\left(\Delta \phi_{\ell-X}=\right.$ $180^{\circ}$ ) like those in $\mathrm{NC}$ and lepton pair events. For events with low hadronic transverse momentum $P_{T}^{X}<1.0 \mathrm{GeV}$, the direction of the hadronic system is not well determined and $\Delta \phi_{\ell-X}$ is set to zero. The quantity $\delta_{\text {miss }}=2 E_{e}^{0}-\sum_{i}\left(E^{i}-P_{z}^{i}\right)$, where the sum runs over all detected particles and $E_{e}^{0}$ is the electron beam energy, gives a measure of the longitudinal balance of the event. For an event where only momentum in the proton direction is undetected, $\delta_{\text {miss }}$ is zero. Further background rejection in the electron channel is achieved using $\zeta_{e}^{2}=4 E_{e} E_{e}^{0} \cos ^{2} \theta_{e} / 2$, where $E_{e}$ is the energy of the final state electron. For $\mathrm{NC}$ events, where the scattered electron is identified as the isolated high transverse momentum electron, $\zeta_{e}^{2}$ is equal to the four momentum transfer squared $Q_{e}^{2}$, as measured by the electron method [18]. The lepton-neutrino transverse mass, $M_{T}^{\ell \nu}$, calculated using the vectors of the missing transverse momentum and the isolated lepton, is used to further 


\begin{tabular}{|c|c|c|}
\hline \multicolumn{3}{|c|}{ H1+ZEUS Isolated Lepton $+P_{T}^{\text {miss }}$ Event Selection } \\
\hline Channel & Electron & Muon \\
\hline $\begin{array}{c}\text { Basic Event } \\
\text { Selection }\end{array}$ & $\begin{array}{c}15^{\circ}<\theta_{\ell} \\
P_{T}^{\ell}>10 \\
P_{T}^{\text {miss }}>1 \\
P_{T}^{\text {calo }}>1\end{array}$ & $\begin{array}{l}<120^{\circ} \\
0 \mathrm{GeV} \\
12 \mathrm{GeV} \\
12 \mathrm{GeV}\end{array}$ \\
\hline Lepton Isolation & $\begin{array}{r}D(\ell ; \text { jet }) \\
D(e ; \text { track })>0.5 \text { for } \theta_{e}>45^{\circ}\end{array}$ & $D(\mu ;$ track $)>0.5$ \\
\hline $\begin{array}{l}\text { Background } \\
\text { Rejection }\end{array}$ & $\begin{array}{c}V_{\mathrm{ap}} / V_{\mathrm{p}} \\
V_{\mathrm{ap}} / V_{\mathrm{p}}<0.15 \text { for } P_{T}^{e}<25 \mathrm{GeV} \\
\Delta \phi_{e-X}<160^{\circ} \\
5<\delta_{\text {miss }}<50 \mathrm{GeV} \\
\zeta_{e}^{2}>5000 \mathrm{GeV}^{2} \text { for } P_{T}^{\text {calo }}<25 \mathrm{GeV} \\
- \\
\quad M_{T}^{\ell \nu}>1 \\
\# \text { electrons }<3\end{array}$ & $\begin{array}{l}<0.5 \\
V_{\text {ap }} / V_{\mathrm{p}}<0.15 \text { for } P_{T}^{\text {calo }}<25 \mathrm{GeV} \\
\Delta \phi_{\mu-X}<170^{\circ} \\
- \\
\quad- \\
10 \mathrm{GeV} \quad \\
P_{T}^{X}>12 \mathrm{GeV} \\
-\end{array}$ \\
\hline
\end{tabular}

Table 1. Selection requirements for the electron and muon channels in the search for events with an isolated lepton and missing transverse momentum.

reject NC (lepton pair) background in the electron (muon) channel.

The lepton polar-angle acceptance, which is the same as that used in the ZEUS publication [5], is the main difference in the event selection with respect to the published $\mathrm{H} 1$ analysis, where isolated leptons are accepted in the range $5^{\circ}<\theta_{\ell}<140^{\circ}$ [6]. Additionally, the more restrictive cuts on $\delta_{\text {miss }}$ and $V_{\mathrm{ap}} / V_{\mathrm{p}}$ are taken from the ZEUS analysis [5]. The minimum lepton-neutrino transverse mass and electron multiplicity requirements are taken from the $\mathrm{H} 1$ analysis [6]. The overall H1(ZEUS) efficiency in the common phase-space analysis to select SM $W \rightarrow e \nu$ events is $30 \%$ (31\%) and to select SM $W \rightarrow \mu \nu$ events is $11 \%$ (9\%), calculated using EPVEC.

The combination of the H1 and ZEUS results is performed by adding both the data and $\mathrm{MC}$ distributions bin by bin. The theoretical uncertainty of $15 \%$ on single $W$ production from the reweighted EPVEC prediction is treated as correlated between the experiments and dominates the SM prediction uncertainty. Dedicated studies of the significant SM background contributions are performed by both experiments, using background-enriched control samples. The systematic uncertainties attributed to the SM background processes are derived from the level of agreement between the data and the SM predictions in these 
control samples. Experimental systematic uncertainties, as well as the uncertainties on the SM background, are treated as uncorrelated between the experiments. The systematic uncertainties determined in the combined analysis are found to be the same as those derived by the individual experiments. A detailed list of the systematic uncertainties considered can be found in the respective publications $[5,6]$.

\section{Results}

The event yields of the combined H1 and ZEUS search for events containing an isolated lepton and missing transverse momentum are summarised in table 2. Results are shown for the electron and muon channels separately as well as combined, for the $e^{+} p$ data, $e^{-} p$ data and the full HERA $e^{ \pm} p$ data. The results are shown for the full selected sample and for a subsample at $P_{T}^{X}>25 \mathrm{GeV}$.

The signal contribution to the SM expectation, dominated by single $W$ production, is $74 \%$ in the combined electron and muon channels for the full HERA $e^{ \pm} p$ data. The H1 and ZEUS parts of the analysis contribute similarly to the total signal expectation. The contribution from signal processes to the total H1 (ZEUS) SM expectation in the electron channel is $76 \%(65 \%)$ and in the muon channel $93 \%(83 \%)$.

In the $e^{+} p$ data, 37 electron events and 16 muon events are observed compared to SM predictions of $38.6 \pm 4.7$ and $11.2 \pm 1.6$ respectively. In the $e^{-} p$ data, 24 electron events and 4 muon events are observed compared to SM predictions of $30.6 \pm 3.6$ and $7.4 \pm 1.1$ respectively. Eleven events in the $\mathrm{H} 1$ publication [6] are not in the common phase space: nine events (eight in the electron channel and one in the muon channel) have $\theta_{\ell}<15^{\circ}$ and two additional electron channel events fail the stricter $\delta_{\text {miss }}$ condition. With respect to the published ZEUS analysis [5], one event is not in the common phase space due to the cut on transverse mass. All twelve events rejected in the combined analysis analysis exhibit $P_{T}^{X}<25 \mathrm{GeV}$.

At large hadronic transverse momentum $P_{T}^{X}>25 \mathrm{GeV}$, a total of 29 events are observed in the complete HERA $e^{ \pm} p$ data compared to a SM prediction of $24.0 \pm 3.2$. In the $e^{+} p$ data alone, where an excess of data over the SM is reported in the H1 analysis [6], 23 events are observed with $P_{T}^{X}>25 \mathrm{GeV}$ compared to a SM prediction of $14.0 \pm 1.9$. Seventeen of these 23 data events are observed in the H1 data, compared to a SM expectation of $6.7 \pm 1.1$.

Figure 1 shows kinematic distributions of the complete HERA $e^{ \pm} p$ data for the combined electron and muon channels. The data are in good agreement with the SM prediction, dominated by single $W$ production. The distribution of the lepton polar angle, $\theta_{\ell}$, shows that the identified lepton is produced mainly in the forward direction. The first bin of the $\Delta \phi_{\ell-X}$ distribution is mainly populated by events with very low values of $P_{T}^{X}$. The shape of the transverse mass $M_{T}^{\ell \nu}$ distribution shows a Jacobian peak as expected from single $W$ production. The observed $P_{T}^{X}, P_{T}^{\text {miss }}$ and $P_{T}^{\ell}$ distributions are also indicative of single $W$ production, where the decay products of the $W$ peak around $40 \mathrm{GeV}$ and the hadronic final state has typically low $P_{T}^{X}$. Figure 2 shows the $P_{T}^{X}$ distribution separately for the combined $e^{+} p$ and $e^{-} p$ data. 
The total and differential single $W$ production cross sections are evaluated bin by bin from the number of observed events, subtracting the number of background events, and taking into account the acceptance and luminosity of the two experiments. The acceptance, defined as the number of $W$ events reconstructed in a bin divided by the number of events generated in that bin, is evaluated using EPVEC and is used to extrapolate the measured cross section to the full phase space. The acceptances for the two experiments are found to be similar in each $P_{T}^{X}$ bin and vary between $27 \%$ and $37 \%$ in the electron channel and between $18 \%$ and $38 \%$ in the muon channel. The purity of the cross section measurement is greater than $70 \%$ in all bins and is also found to behave similarly for the two experiments. For $P_{T}^{X}<12 \mathrm{GeV}$, the electron measurement is used to estimate the muon cross section under the assumption of lepton universality. Leptonic tau decays from $W \rightarrow \tau \nu$ events are taken into account in the cross section calculation. The cross sections are quoted at the luminosity-weighted mean centre-of-mass energy $\sqrt{s}=317 \mathrm{GeV}$ of the complete HERA data.

The total single $W$ boson production cross section at HERA is measured as:

$$
1.06 \pm 0.16 \text { (stat.) } \pm 0.07 \text { (sys.) pb, }
$$

which agrees well with the SM prediction of $1.26 \pm 0.19 \mathrm{pb}$. The measured differential cross sections, in bins of $P_{T}^{X}$, are shown in figure 3 and given in table 3 . The differential cross section agrees well with the SM prediction.

\section{Conclusions}

A search for events containing an isolated electron or muon and large missing transverse momentum produced in $e^{ \pm} p$ collisions is performed with the H1 and ZEUS detectors at HERA in a common phase space. The full HERA $e^{ \pm} p$ high energy data sample from both experiments is analysed, corresponding to a total integrated luminosity of $0.98 \mathrm{fb}^{-1}$. A total of 81 events are observed in the data, compared to a SM prediction of $87.8 \pm 11.0$. In the $e^{+} p$ data, at large hadronic transverse momentum $P_{T}^{X}>25 \mathrm{GeV}$, a total of 23 data events are observed compared to a SM prediction of $14.0 \pm 1.9$. The total and differential single $W$ production cross sections are measured and are found to be in agreement with the SM predictions.

\section{Acknowledgments}

We are grateful to the HERA machine group whose outstanding efforts have made these experiments possible. We appreciate the contributions to the construction and maintenance of the $\mathrm{H} 1$ and ZEUS detectors of many people who are not listed as authors. We thank our funding agencies for financial support, the DESY technical staff for continuous assistance and the DESY directorate for their support and for the hospitality they extended to the non-DESY members of the collaborations. 


\begin{tabular}{|c|c|c|c|c|c|c|c|c|c|c|c|c|}
\hline \multirow{2}{*}{\multicolumn{2}{|c|}{$\begin{array}{l}\text { H1+ZEUS } \\
1994-2007 e^{+} p \\
\text { Electron }\end{array}$}} & \multirow{2}{*}{$\frac{0.59 \mathrm{fb}^{-1}}{\text { Total }}$} & \multirow{2}{*}{$\begin{array}{c}\text { Data } \\
37\end{array}$} & \multicolumn{3}{|c|}{$\begin{array}{c}\text { SM } \\
\text { Expectation }\end{array}$} & \multicolumn{3}{|c|}{$\begin{array}{c}\text { SM } \\
\text { Signal }\end{array}$} & \multicolumn{3}{|c|}{$\begin{array}{l}\text { Other SM } \\
\text { Processes }\end{array}$} \\
\hline & & & & 38.6 & \pm & 4.7 & 28.9 & \pm & 4.4 & 9.7 & \pm & 1.4 \\
\hline & & $>25 \mathrm{GeV}$ & 12 & 7.4 & \pm & 1.0 & 6.0 & \pm & 0.9 & 1.5 & \pm & 0.3 \\
\hline \multirow[t]{2}{*}{ Muon } & & Total & 16 & 11.2 & \pm & 1.6 & 9.9 & \pm & 1.6 & 1.3 & \pm & 0.3 \\
\hline & $P_{T}^{X}$ & $>25 \mathrm{GeV}$ & 11 & 6.6 & \pm & 1.0 & 5.9 & \pm & 0.9 & 0.8 & \pm & 0.2 \\
\hline \multirow[t]{2}{*}{ Combined } & & Total & 53 & 49.8 & \pm & 6.2 & 38.8 & \pm & 5.9 & 11.1 & \pm & 1.5 \\
\hline & & $>25 \mathrm{GeV}$ & 23 & 14.0 & \pm & 1.9 & 11.8 & \pm & 1.9 & 2.2 & \pm & 0.4 \\
\hline
\end{tabular}

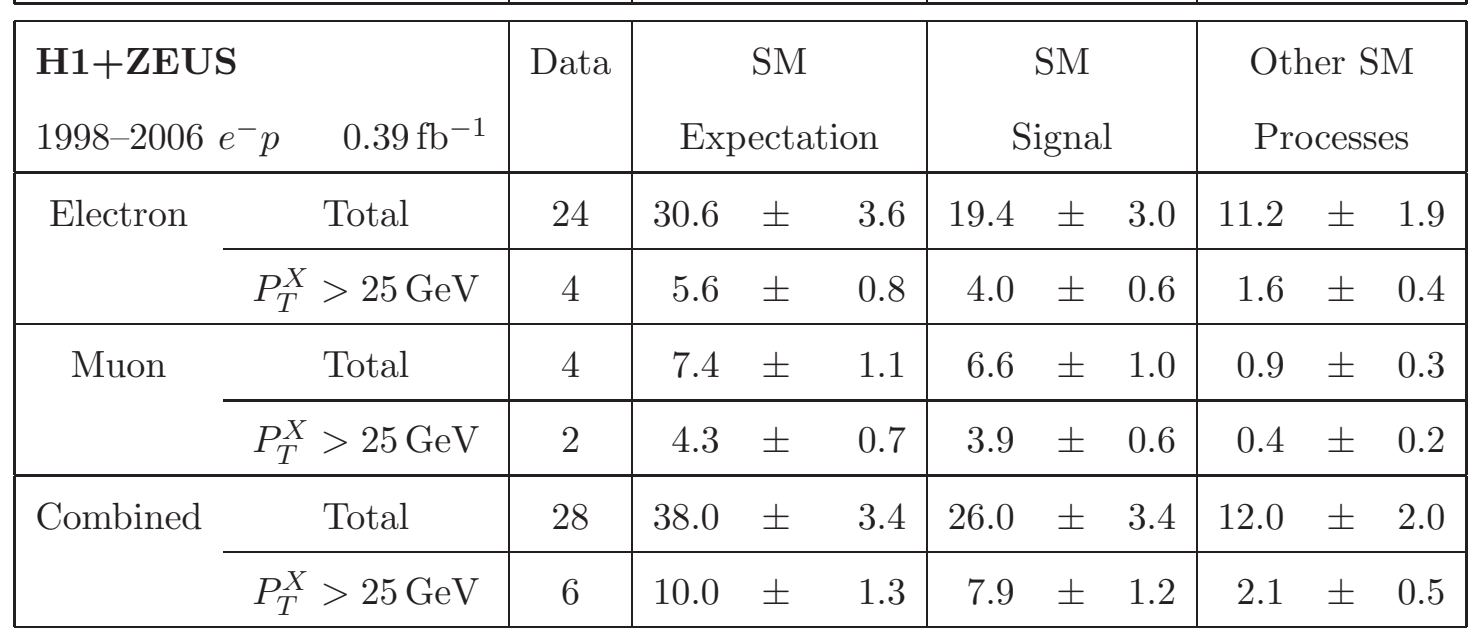

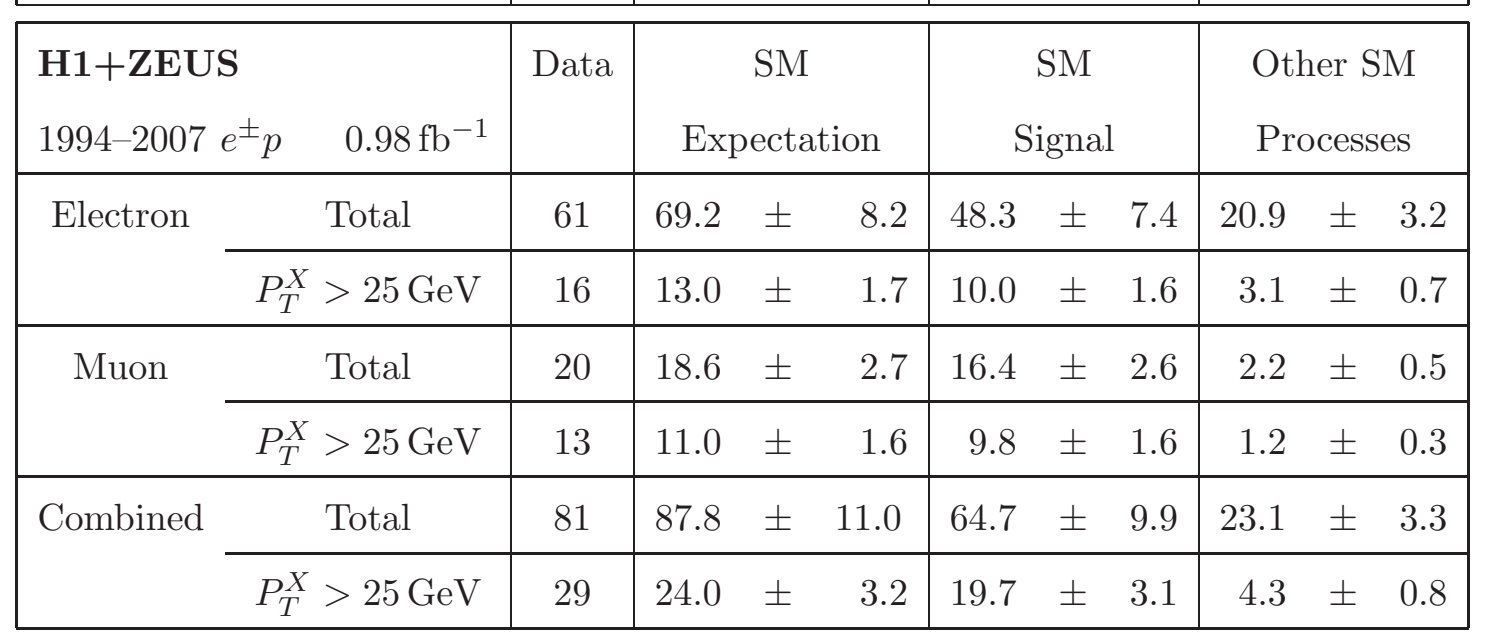

Table 2. Summary of the combined H1 and ZEUS search for events with an isolated electron or muon and missing transverse momentum for the $e^{+} p$ data (top), $e^{-} p$ data (middle) and the full HERA data set (bottom). The results are shown for the full selected sample and for the subsample with hadronic transverse momentum $P_{T}^{X}>25 \mathrm{GeV}$. The number of observed events is compared to the SM prediction. The SM signal (dominated by single $W$ production) and the total background contribution are also shown. The quoted uncertainties contain statistical and systematic uncertainties added in quadrature. 


\begin{tabular}{|c|c|c|}
\hline \multicolumn{3}{|c|}{ H1+ZEUS Differential Single $\boldsymbol{W}$ Production Cross Section } \\
\hline$P_{T}^{X}[\mathrm{GeV}]$ & Measured \pm stat. \pm sys. $[\mathrm{fb} / \mathrm{GeV}]$ & SM NLO $[\mathrm{fb} / \mathrm{GeV}]$ \\
\hline $0-12$ & $33.6 \pm 12.3 \pm 5.0$ & $62.7 \pm 9.4$ \\
$12-25$ & $20.6 \pm 6.0 \pm 1.9$ & $20.7 \pm 3.1$ \\
$25-40$ & $12.7 \pm 3.6 \pm 1.0$ & $9.8 \pm 1.5$ \\
$40-100$ & $2.1 \pm 0.7 \pm 0.2$ & $1.5 \pm 0.2$ \\
\hline
\end{tabular}

Table 3. The differential single $W$ boson production cross section $\mathrm{d} \sigma_{W} / \mathrm{d} P_{T}^{X}$, with statistical (stat.) and systematic (sys.) errors, measured using the combined H1 and ZEUS data. The cross sections are quoted at a centre-of-mass energy $\sqrt{s}=317 \mathrm{GeV}$. Also shown are the expectations, including the theoretical uncertainties, for the Standard Model calculated at next-to-leading order (SM NLO). 


\section{Events with an Isolated Lepton and $\mathrm{P}_{\mathrm{T}}^{\text {miss }}$ at HERA}

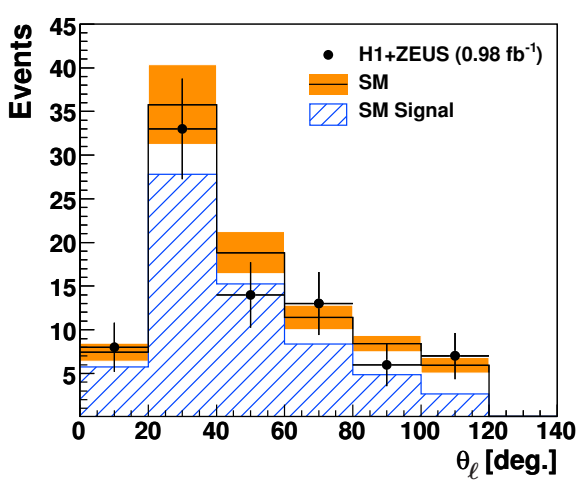

(a)

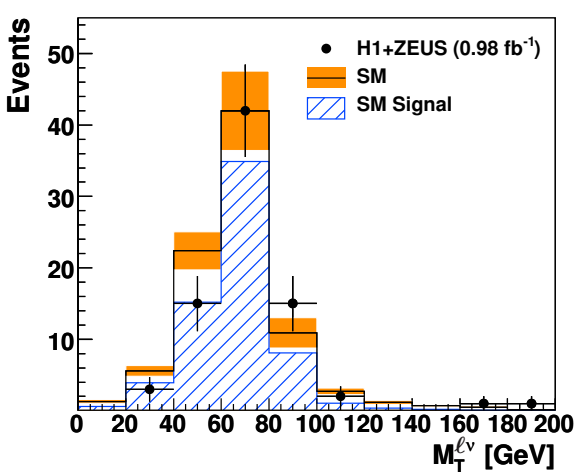

(c)

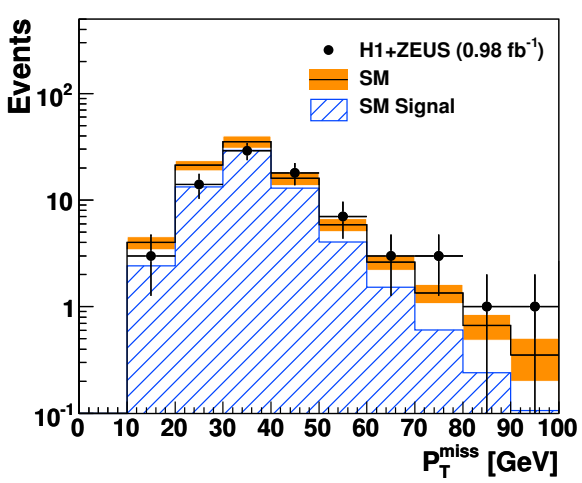

(e)

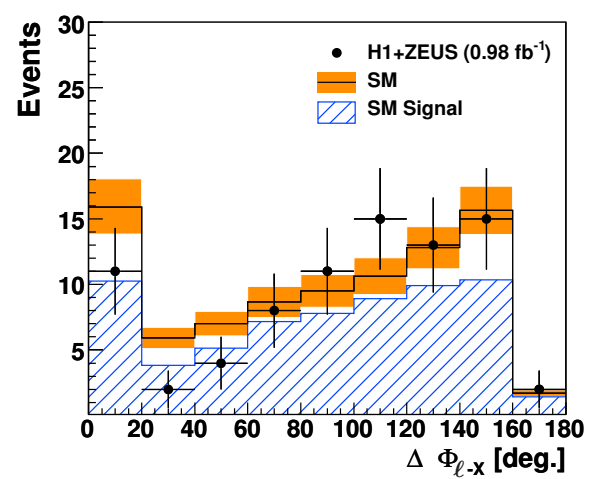

(b)

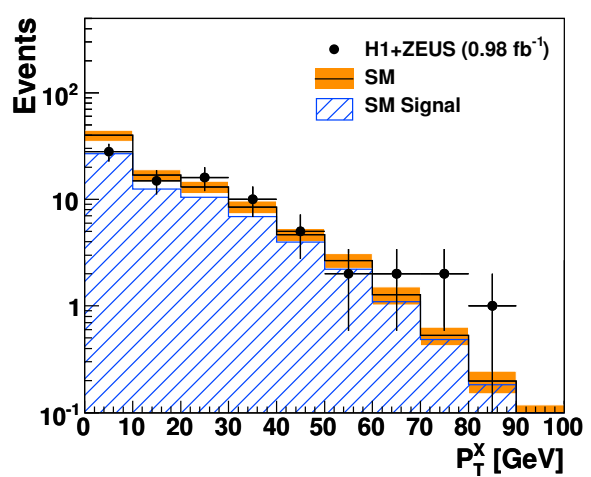

(d)

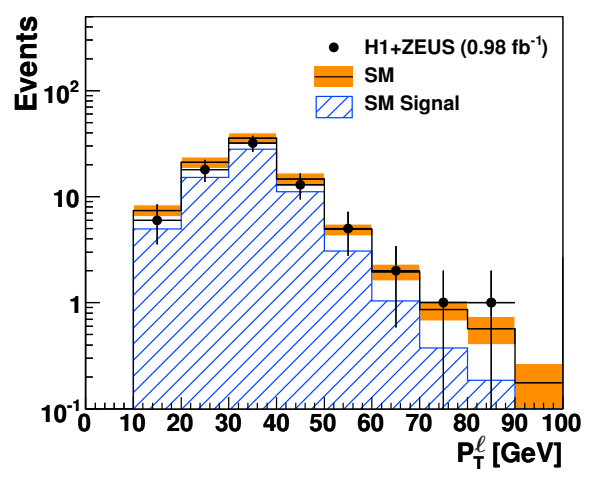

(f)

Figure 1. Distributions of kinematic variables of events with an isolated electron or muon and missing transverse momentum in the full HERA $e^{ \pm} p$ data. Shown are: the polar angle of the lepton $\theta_{\ell}(\mathrm{a})$, the difference in the azimuthal angle of the lepton and the hadronic systems $\Delta \phi_{\ell-X}(\mathrm{~b})$, the lepton-neutrino transverse mass $M_{T}^{\ell \nu}(\mathrm{c})$, the hadronic transverse momentum $P_{T}^{X}(\mathrm{~d})$, the missing transverse momentum $P_{T}^{\text {miss }}$ (e) and the transverse momentum of the lepton $P_{T}^{\ell}$ (f). The data (points) are compared to the SM expectation (open histogram). The signal component of the SM expectation, dominated by single $W$ production, is shown as the hatched histogram. The total uncertainty on the SM expectation is shown as the shaded band. 


\section{Events with an Isolated Lepton and $\mathrm{P}_{\mathrm{T}}^{\text {miss }}$ at HERA}

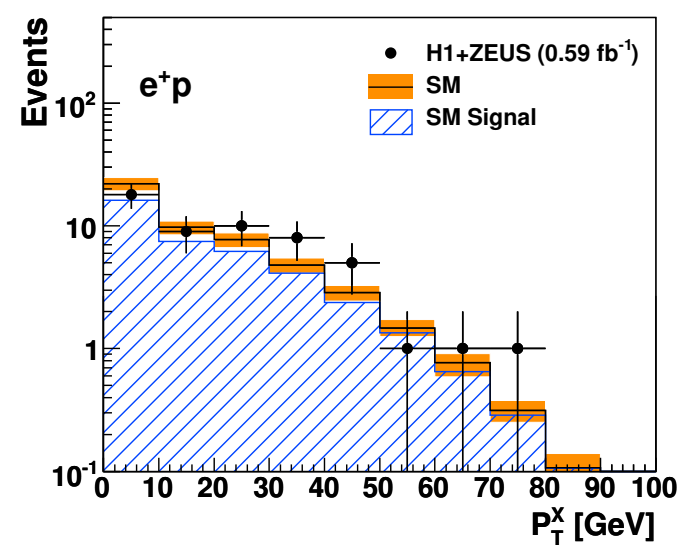

(a)

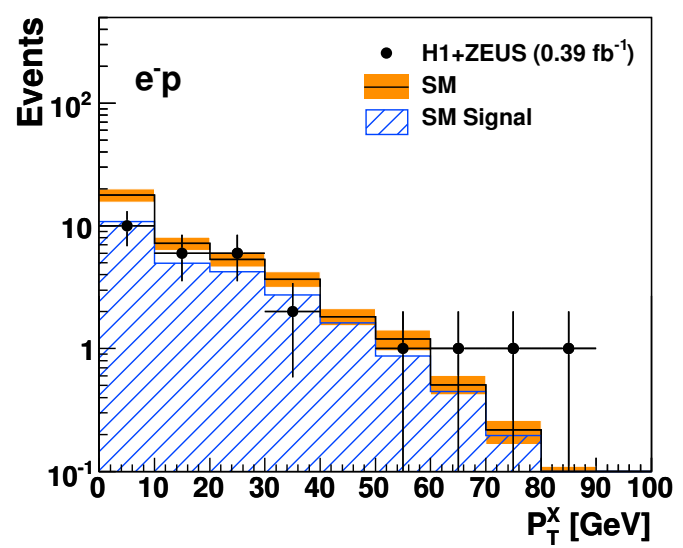

(b)

Figure 2. Distributions of the hadronic transverse momentum $P_{T}^{X}$ of events with an isolated electron or muon and missing transverse momentum for the $e^{+} p$ (a) and $e^{-} p$ (b) HERA data. The data (points) are compared to the SM expectation (open histogram). The signal component of the SM expectation, dominated by single $W$ production, is shown as the hatched histogram. The total uncertainty on the SM expectation is shown as the shaded band.

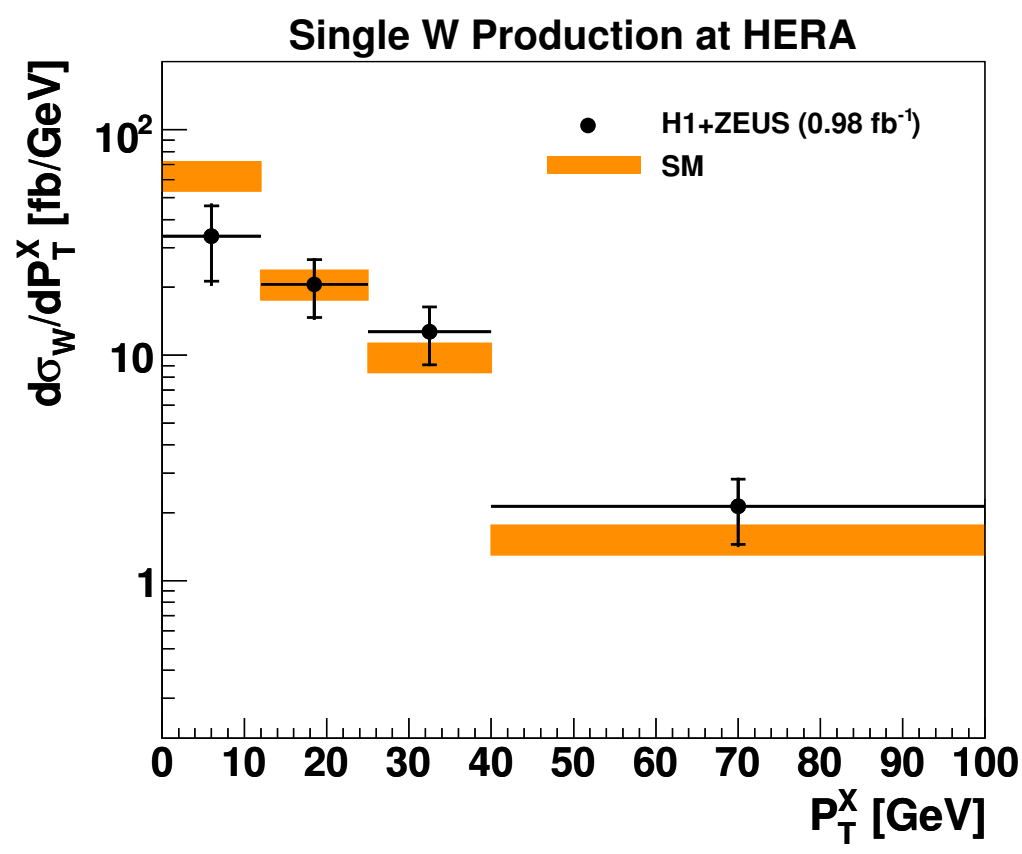

Figure 3. The single $W$ production cross section as a function of the hadronic transverse momentum, $P_{T}^{X}$, measured using the combined H1 and ZEUS data at a centre-of-mass energy of $\sqrt{s}=317 \mathrm{GeV}$. The inner error bar represents the statistical error and the outer error bar indicates the statistical and systematic uncertainties added in quadrature. The shaded band represents the uncertainty on the SM prediction. 
Open Access. This article is distributed under the terms of the Creative Commons Attribution Noncommercial License which permits any noncommercial use, distribution, and reproduction in any medium, provided the original author(s) and source are credited.

\section{References}

[1] H1 collaboration, C. Adloff et al., Observation of events with an isolated high energy lepton and missing transverse momentum at HERA, Eur. Phys. J. C 5 (1998) 575 [hep-ex/9806009] [SPIRES].

[2] ZEUS collaboration, J. Breitweg et al., W production and the search for events with an isolated high-energy lepton and missing transverse momentum at HERA, Phys. Lett. B 471 (2000) 411 [hep-ex/9907023] [SPIRES].

[3] H1 collaboration, V. Andreev et al., Isolated electrons and muons in events with missing transverse momentum at HERA, Phys. Lett. B 561 (2003) 241 [hep-ex/0301030] [SPIRES].

[4] ZEUS collaboration, S. Chekanov et al., Search for single-top production in ep collisions at HERA, Phys. Lett. B 559 (2003) 153 [hep-ex/0302010] [SPIRES].

[5] ZEUS collaboration, S. Chekanov et al., Search for events with an isolated lepton and missing transverse momentum and a measurement of $W$ production at HERA, Phys. Lett. B 672 (2009) 106 [arXiv:0807.0589] [SPIRES].

[6] H1 collaboration, F.D. Aaron et al., Events with isolated leptons and missing transverse momentum and measurement of $W$ production at HERA, Eur. Phys. J. C 64 (2009) 251 [arXiv: 0901.0488] [SPIRES].

[7] H1 collaboration, I. Abt et al., The H1 detector at HERA, Nucl. Inst. Meth. A 386 (1997) 310.

[8] H1 collaboration, I. Abt et al., The tracking, calorimeter and muon detectors of the H1 experiment at HERA, Nucl. Inst. Meth. A 386 (1997) 348.

[9] H1 SPACAL Group, R.D. Appuhn et al., The H1 lead/scintillating-fibre calorimeter, Nucl. Instrum. Meth. A 386 (1997) 397 [SPIRES].

[10] ZEUS collaboration, U. Holm, The ZEUS Detector, status report, unpublished, DESY (1993), available at http://www-zeus.desy.de/bluebook/bluebook.html.

[11] U. Baur, J.A.M. Vermaseren and D. Zeppenfeld, Electroweak vector boson production in high-energy ep collisions, Nucl. Phys. B 375 (1992) 3 [SPIRES].

[12] K.-P.O. Diener, C. Schwanenberger and M. Spira, Photoproduction of $W$ bosons at HERA: QCD corrections, Eur. Phys. J. C 25 (2002) 405 [hep-ph/0203269] [SPIRES].

[13] P. Nason, R. Ruckl and M. Spira, A note on $W$ boson production at HERA, J. Phys. G 25 (1999) 1434 [hep-ph/9902296] [SPIRES].

[14] M. Spira, $W$ boson production at NLO, hep-ph/9905469 [SPIRES].

[15] S.D. Ellis and D.E. Soper, Successive combination jet algorithm for hadron collisions, Phys. Rev. D 48 (1993) 3160 [hep-ph/9305266] [SPIRES].

[16] S. Catani, Y.L. Dokshitzer, M.H. Seymour and B.R. Webber, Longitudinally invariant $K_{t}$ clustering algorithms for hadron hadron collisions, Nucl. Phys. B 406 (1993) 187 [SPIRES].

[17] H1 collaboration, C. Adloff et al., Measurement of neutral and charged current cross-sections in positron proton collisions at large momentum transfer, Eur. Phys. J. C 13 (2000) 609 [hep-ex/9908059] [SPIRES].

[18] F. Jacquet and A. Blondel, Detectors for charged current events, proceedings of Study of an ep Facility for Europe, U. Amaldi ed., DESY (1979). 